\title{
Structures of bak with lipids: implications for pore formation
}

\author{
Peter Czabotar ${ }^{1}$, Angus Cowan ${ }^{1}$, Peter Colman ${ }^{1}$ \\ ${ }^{1}$ Structural Biology, Walter And Eliza Hall Institute Of Medical Research, Melbourne, Australia \\ E-mail: czabotar@wehi.edu.au
}

Apoptosis is a process of altruistic cell suicide critical for the development and maintenance of homeostasis in mulitcellular organisms, the $\mathrm{Bcl}-2$ family of proteins play a key role in the regulation of apoptosis via the intrinsic pathway. A critical step in the pathway is the permeabilisation of the outer mitochondrial membrane by the Bcl-2 family effector proteins Bak and Bax, this results in the release of apoptogenic factors that initiate a cascade of signaling leading ultimately to the death of the cell. The events preceding permeabilisation, including activation of Bak and Bax and formation of symmetric homodimers, have been well characterised by structural and biochemical studies (eg $[1,2])$. What is currently lacking is a clear picture of how these Bak and Bax homodimers interact with the membrane and self-associate into large membrane-permeabilising oligomers. We have solved several structures of the dimerisation or "core" domain of Bak in complex with detergents and lipids, to our knowledge these are the first examples of such complexes involving Bcl-2 family proteins. The structures have implications for Bak oligomerisation, as well as membrane destabilisation and pore formation by Bak oligomers.

[1] Czabotar, P. E., et al. (2013). Cell, 152, 519-531

[2] Brouwer, J. M., et al. (2014). Mol Cell, 55, 938-946

Keywords: Cell Death, Apoptosis, Lipid-Protein Interactions 